\title{
Photonics for new challenges
}

\author{
Bogdan B. Kosmowski* \\ Faculty of Electronics, Telecommunications and Informatics, Gdansk University of Technology, Narutowicza 11, \\ 80-233 Gdańsk
}

Received June 28, 2011; accepted June 29, 2011; published June 30, 2011

\begin{abstract}
A brief editorial overview of the current special issue of the Photonics Letters of Poland is presented. This special issue is devoted to "Photonics for new challenges". It contains 15 carefully selected papers covering recent developments in areas ranging from photonic materials and components through applications of photonic metrology, to the technologies enabling the Petabit Photonic Internet.
\end{abstract}

It is a great honor and pleasure to present another special issue of the Photonics Letters of Poland to our readers. This special issue is dedicated to "Photonics for new challenges".

Photonics as a new and promising field of science and technology creates an enormous application potential. Its dynamic growth is expectantly watched by scientists, engineers and professionals from all fields of science, technology, and other human activities, who can translate discoveries and developments in photonics into progress in their fields of activity.

These discoveries, which involve complex interactions of photons with matter and the environment, often in the presence of electric or magnetic fields, can frequently be described only in terms of quantum mechanics. As a result, for the first time in history, people started to look at and design materials at the nanoscale level, creating structures, such as quantum wells or dots, or materials with a negative refractive index, whose properties and functions cannot be obtained in any other way.

All this research has created new opportunities in investigating and monitoring materials and objects of almost any kind, in encoding, transmitting, and visualizing information. It also opened up a totally new era in material science whose products will exhibit properties sometimes totally unrelated to those of bulk materials.

Therefore, PHOTONICS is a gateway to a fascinating world in which photons will work (if we can press them into doing that!) fulfilling new tasks and performing new functional properties.

\footnotetext{
*E-mail: bogdan.kosmowski@eti.pg.gda.pl
}

The papers of this issue of the Photonics Letters of Poland describe selected recent developments in all key areas of photonics - from innovative photonic components and photonic materials, through investigation of their properties and optimization of their manufacturing technologies, to applications of photonic metrology and to the Petabit Photonic Internet.

Having unique properties, Quantum Cascade Lasers (QCLs) are subject of intense research, aimed at modifying their characteristics (paper by Konupek et al.) and thermal issues (papers by Sarzała et al. and by Bugajski et al.).

Novel types of photodetectors are also of interest well beyond photonics as e.g. InGaSb photodiodes, which are discussed in the paper by Tanzid et al.

New photonic technologies which form the basis for manufacturing an ever-expanding range of photonic components are the subject of study, optimization of their properties and methods of precise monitoring.

Examples of such research on new technologies are:

- application of the sol-gel technique for creating sol-gel films for active coating devices (paper of Kościelska et al.);

- study on the electroabsorption of layers of bathocuproine, material for organic electroluminescent elements OLED (paper by Stompor);

- study of luminescence of new materials and in very specific conditions (papers of Lazarowska et al.)

- a very interesting paper for many applications "Carbon Nitride films deposited by DC magnetron sputtering" (Hippler et al.),

- and a subtle analysis to obtain the assumed characteristics of Thin Film Filters (Frühauf et al.).

The purpose of photonic measurement circuits and systems is to obtain accurate and reliable information on a wide variety of classes of objects and their characteristics. Recently, a very intensively developed domain of application of the $\mathrm{THz}$ radiation is an example of the photonic success in meeting important social needs for national security. The basis for such systems is information about the spectral properties of materials in the $\mathrm{THz}$ range (Palka et al.). 
The responsibility of society is to take care of cultural heritage and therefore of the safety of such unique historic objects, the conditions of conservation projects and their progress assessment should be very precisely defined, (Zmuda-Trzebiatowska et al.)

Modern measurement methods, such as OCT (Optical Coherence Tomography), allow us, often in very difficult conditions, to measure the characteristics of phenomena or objects in a direct way - such as suspension viscosity done by Doppler OCT (paper by Lauri et al.).

Photonic measurement methods are quite versatile, being used in various applications, as exemplified by the study of Electrokinetic mobility of microparticles by holografic microscopy (paper of Haapalainen et al.).

New Liquid Crystal Based Light steering Optical Elements are the subject of the paper by Valyukh.

The feasibility of creating a complex function is epitomized by sophisticated photonic systems such as the Photonic Internet System, which encircles the world and ensures the transfer of information between individual participants in the network located almost anywhere on Earth.

The problems to be solved for the Internet of the Future are: extension of the range, increase of availability for an increasing number of participants, the requirement for higher information transfer rate, which are discussed in the paper about "Petabit Photonic Internet" by Romaniuk.

The papers selected for and presented in this issue describe some recent developments in all key areas of photonics. They illustrate new developments, trends and research results in photonics. They also show the ongoing search for new solutions of the problems in the rapidly changing world, in which photons play a fundamental role.

We would like to express our gratitude to the authors for their valuable contributions, reviewers for thorough and prompt review, and to the Editorial Team for their diligent and dedicated work and involvement in preparing this special issue. 